\section{(C) OPEN ACCESS}

\title{
Primary percutaneous coronary intervention for ST elevation myocardial infarction in nonagenarians
}

\author{
Thibaut Petroni, ${ }^{1}$ Azfar Zaman, ${ }^{2}$ Jean-Louis Georges, ${ }^{3}$ Nadjib Hammoudi, ${ }^{1}$ \\ Emmanuel Berman, ${ }^{1}$ Amit Segev, ${ }^{4}$ Jean-Michel Juliard, ${ }^{5}$ Olivier Barthelemy, ${ }^{1}$ \\ Johanne Silvain, ${ }^{1}$ Rémi Choussat, ${ }^{1}$ Claude Le Feuvre, ${ }^{1}$ Gérard Helft ${ }^{1}$
}

\begin{abstract}
${ }^{1}$ Cardiology Institute, PitiéSalpêtrière Hospital, UPMC, APHP, Paris, France

${ }^{2}$ Freeman Hospital and Institute of Cellular Medicine, Newcastle University, Newcastle Upon Tyne, UK

${ }^{3}$ Centre Hospitalier de Versailles, André Mignot Hospital, Le Chesnay, France ${ }^{4}$ Heart Institute, Chaim Sheba Medical Center, Tel-Hashomer, Israël

${ }^{5}$ Bichat Hospital, APHP, Paris, France
\end{abstract}

\section{Correspondence to} Professor Gérard Helft, Cardiology Institute, PitiéSalpêtrière Hospital, 47-83, boulevard de I'hôpital, Paris 75013, France;

gerard.helft@psl.aphp.fr

Received 26 October 2015 Revised 28 April 2016 Accepted 29 April 2016 Published Online First 13 July 2016

\section{CrossMark}

\author{
To cite: Petroni $\mathrm{T}$, \\ Zaman A, Georges J-L, et al. \\ Heart 2016;102
}

1648-1654.

\begin{abstract}
Objective To assess outcomes following primary percutaneous coronary intervention (PCI) for ST-segment elevation acute myocardial infarction (STEMI) in nonagenarian patients.

Methods We conducted a multicentre retrospective study between 2006 and 2013 in five international highvolume centres and included consecutive all-comer nonagenarians treated with primary PCI for STEMI. There were no exclusion criteria. We enrolled 145 patients and collected demographic, clinical and procedural data. Severe clinical events and mortality at 6 months and 1 year were assessed.
\end{abstract}

Results Cardiogenic shock was present at admission in $21 \%$. Median (IQR) delay between symptom onset and balloon was $3.7(2.4-5.6)$ hours and $60 \%$ of procedures were performed through the transradial approach. Successful revascularisation of the culprit vessel was obtained in $86 \%$ of the cases (thrombolysis in myocardial infarction flow of 2 or 3). Major or clinically relevant bleeding was observed in $4 \%$ of patients. Median left ventricular ejection fraction post $\mathrm{PCl}$ was $41.5 \%$ (32.0-50.0). The in-hospital mortality was $24 \%$, with 6 months and 1-year survival rates of $61 \%$ and $53 \%$, respectively.

Conclusions In our study, primary $\mathrm{PCl}$ in nonagenarians with STEMI was achieved and feasible through a transradial approach. It is associated with a high rate of reperfusion of the infarct-related artery and $53 \%$ survival at 1 year. These results suggest that primary $\mathrm{PCl}$ may be offered in selected nonagenarians with acute myocardial infarction.

\section{INTRODUCTION}

Medical and technical progress has led to increased life expectancy, resulting in a significant proportion of very old patients with specific health problems. In this growing population, cardiovascular disease is frequent and ischaemic heart disease remains one of the leading causes of morbidity and mortality. ${ }^{12}$ However, most clinical trials exclude patients aged $>75-80$ years as prolonged follow-up may be compromised by limited life expectancy. It also appears that side effects of new therapies are mostly observed in patients aged $>75$ years. ${ }^{3}$ Other factors such as atypical symptoms, delayed presentation and associated comorbidities further add to advanced age as being one of the most powerful predictors of adverse outcome in acute coronary syndromes. ${ }^{4}$ As a result, elderly patients $>75$ years old are under-represented in clinical trials. ${ }^{5-8}$ Additionally, prior studies have reported an increased rate of vascular complications, bleeding and cardiac mortality associated with percutaneous coronary intervention (PCI) in the elderly. ${ }^{9-11}$ As a consequence, it is difficult to assess the risk/benefit balance for care protocols and pharmacological interventions in these patients. ${ }^{12}$ To date, no clinical randomised trial is available for the management of ST-segment elevation myocardial infarction (STEMI) in elderly patients. Non-revascularised patients admitted with STEMI are known to carry poor outcomes. ${ }^{13}{ }^{14}$ Even though primary PCI has been established as the standard of care, a conservative strategy is often adopted when facing this situation in nonagenarians. ${ }^{15} 16$ The first case report of primary $\mathrm{PCI}$ in a nonagenarian was published in 2002. ${ }^{17}$ Few studies based on cohorts have suggested feasibility for invasive management of STEMI in nonagenarians. ${ }^{18-21}$ Our team has recently published the outcome of the largest series of nonagenarians presenting with STEMI, but data are limited to in-hospital follow-up only. ${ }^{22}$ Through this paper, we present data of the largest series of consecutive patients aged 90 years or older admitted with STEMI and treated by primary PCI in contemporary practice.

\section{METHODS}

\section{Study population}

We retrospectively enrolled 145 consecutive patients aged $\geq 90$ years hospitalised for STEMI and treated with primary PCI in five international high-volume centres between 2006 and 2013. These centres were Freeman hospital (Newcastle Upon Tine, UK), Pitié-Salpêtrière teaching hospital (Paris, France), Chaim-Sheba Medical Center (Tel-Hashomer, Israel), André Mignot hospital (Versailles, France) and Bichat teaching hospital (Paris, France) and respectively provided 46, 43, 28, 20 and 8 patients. Their respective numbers of primary PCI in 2013 were 250, 350, 290, 112 and 200. All patients had follow-up during in-hospital stay, with completed baseline characteristics, pharmacological management, angiographic findings and procedure results. A 12-month follow-up was obtained during patients' visit to outpatient clinics or by telephone interview. The inclusion criteria for primary PCI were clinical symptoms (such as general weakness, thoracic symptoms, eg, persisting chest pain over $30 \mathrm{~min}$ or dyspnoea, digestive 
symptoms such as nausea or vomiting, neurological alteration including acute confusional state) combined with electrocardiographic ST-segment elevation over $1 \mathrm{mV}$ in at least two contiguous leads and admission within 12 hours of symptoms onset or 24 hours for patients with evidence of continuing ischaemia. There were no exclusion criteria.

\section{Cardiac catheterisation}

The interventional procedure was performed by senior interventional cardiologists using 6 Fr guiding catheters in all cases once verbal consent for treatment had been obtained from the patient or relatives. The vast majority of patients received a loading dose of two antiplatelet therapies and heparin before PCI. The choice of antithrombotic therapy, the use of thrombus aspiration catheter and the type of stent were left to the discretion of the attending interventionist.

\section{Outcomes evaluation}

All patients were observed in a coronary care unit for at least 24 hours before discharge or transfer. We recorded the incidence of severe clinical events such as death, recurrent myocardial infarction, target vessel revascularisation, stroke, procedure-related complications, acute renal failure and major or clinically relevant bleeding during hospitalisation. Survival data at 6 months and 1 year were collected. A successful procedure was defined as achievement of thrombolysis in myocardial infarction (TIMI) grade 2 or 3 flow, $<50 \%$ residual stenosis and absence of any major clinical adverse event within 24 hours. A failed procedure was defined as resulting in TIMI grade 0-1 flow regardless of residual stenosis with the absence of any major clinical adverse event within 24 hours. This could result from no reflow phenomenon or coronary dissection. A complicated procedure was defined as resulting in death, recurrent myocardial infarction (ie, at least doubling creatine kinase from the initial value with $>10 \%$ of creatine kinase-MB fraction (CK-MB)) within 24 hours. Major and minor bleeding events were defined using the criteria of the TIMI trial group and acute renal failure was defined as an absolute increase in serum creatinine of at least $0.3 \mathrm{mg} / \mathrm{dL}(26.4 \mu \mathrm{mol} / \mathrm{L})$ or a percentage increase in serum creatinine of at least $50 \%$ according to the criteria.

\section{Statistical analysis}

Continuous variables are expressed as median and IQR, and discrete variables as absolute values and percentages. The log-rank test was used to compute the significance of time-to-event data, and survival rates were estimated using the Kaplan-Meier method. The Kaplan-Meier curve was plotted through day 730 (2 years). An additional Cox proportional hazard regression analysis was performed to describe the effects of gender and age on survival, with and without adjustment on centre. Analyses were performed using SPSS software V.23 (IBM, Armonk, New York, USA).

\section{RESULTS}

\section{Patient demographics}

Median (IQR) age was 92 (91-94) years (extreme 90-102) with the majority female (62\%) and associated comorbidities were low. Baseline characteristics of the study population are reported in table 1 , and biological parameters are summarised in table 2 .

In this cohort, no patient was admitted for asymptomatic acute myocardial infarction. The clinical presentation of these patients was severe with $43 \%$ in Killip class II-IV including Risk, Injury, Failure, Loss and End-Stage Kidney Disease

Table 1 Baseline demographics and clinical data

\begin{tabular}{|c|c|}
\hline \multicolumn{2}{|l|}{ Baseline characteristics } \\
\hline Age (years) & $92.0(91.0-94.0)$ \\
\hline Sex ratio ( $\%$ men) & 38 \\
\hline Body mass index $\left(\mathrm{kg} / \mathrm{m}^{2}\right)$ & $23.4(21.0-25.5)$ \\
\hline Hypertension (\%) & 53 \\
\hline Hyperlipidaemia (\%) & 25 \\
\hline Diabetes mellitus (\%) & 14 \\
\hline Active smoker (\%) & 8 \\
\hline \multicolumn{2}{|l|}{ History of coronary heart disease (\%) } \\
\hline Previous MI & 24 \\
\hline Previous $\mathrm{PCl}$ & 4 \\
\hline Previous CABG & 5 \\
\hline \multicolumn{2}{|l|}{ Clinical presentation (\%) } \\
\hline Chest pain & 86 \\
\hline Dyspnoea & 21 \\
\hline \multicolumn{2}{|l|}{ Killip class at admission (\%) } \\
\hline 1 & 57 \\
\hline II & 12 \\
\hline III & 10 \\
\hline IV & 21 \\
\hline LVEF at admission (\%) & $41.5(32.0-50.0)$ \\
\hline \multicolumn{2}{|l|}{ Electrocardiographic presentation (\%) } \\
\hline Anterior STEMI location & 39 \\
\hline LBBB & 4 \\
\hline STEMI with ST elevation in avR & 3 \\
\hline
\end{tabular}

nearly a third presenting with cardiogenic shock or severe pulmonary oedema.

\section{Antithrombotic management}

Antithrombotic management of the patients is recorded in table 3. All patients received an intravenous loading dose of aspirin between 250 and $500 \mathrm{mg}$ before the procedure. P2Y12 inhibitors were administered in $97 \%$ of the cases and consisted majorly in clopidogrel loading (300-600 mg). Glycoprotein IIb/ IIIa inhibitors, predominantly abciximab, were administered during the cardiac catheterisation in $36 \%$ of the patients. Heparin, predominantly unfractionated heparin, was used in

Table 2 Biological parameters

\begin{tabular}{lc}
\hline Biological data & \\
\hline Cardiac troponin (cTnl) peak (ng/mL) & $45.0(10.9-94.0)$ \\
Creatine kinase peak (UI/L) & $932.0(111.5-2468.0)$ \\
Haemoglobin at admission (g/dL) & $11.9(11.0-12.8)$ \\
Nitrogen urea at admission (mmol/L) & $11.9(6.7-15.2)$ \\
Serum creatinine at admission ( $\mu \mathrm{mol} / \mathrm{L})$ & $111.0(89.0-139.7)$ \\
Creatinine clearance (mL/min)_-MDRD & $49.0(36.8-61.0)$ \\
Cholesterol (g/L) & $1.66(1.43-1.94)$ \\
LDL-C (g/L) & $0.92(0.72-1.09)$ \\
HDL-C (g/L) & $0.52(0.42-0.65)$ \\
Triglyceride (mmol/L) & $0.82(0.66-1.05)$ \\
Fibrinogen (g/L) & $4.1(3.5-5.0)$ \\
\hline HDL-C, high-density lipoprotein cholesterol; LDL-C, low-density lipoprotein cholesterol. \\
MDRD, Modification of the Diet in Renal Disease.
\end{tabular}


Table 3 Antithrombotic therapy pre or per $\mathrm{PCl}$

\begin{tabular}{lr}
\hline Antithrombotic treatment & \\
\hline Aspirin (\%) & 99 \\
Clopidogrel (\%) & 94 \\
Prasugrel (\%) & 3 \\
Ticagrelor (\%) & 0 \\
Glycoprotein Ilbllla inhibitors (\%) & 36 \\
Heparin (\%) & 90 \\
LMWH (\%) & 23 \\
Bivalirudin (\%) & 1 \\
\hline LMWH, low-molecular weight heparin PCl, percutaneous coronary intervention &
\end{tabular}

$90 \%$ of cases. Unfractionated heparin was administered at $100 \mathrm{UI} / \mathrm{kg}$ before the beginning of the procedure with additional doses at the discretion of the physician. When chosen, low-molecular-weight heparin was administered at an intravenous loading dose of $0.5 \mathrm{mg} / \mathrm{kg}$, then additional doses if necessary depending on procedure duration.

\section{Angiographic and procedural characteristics}

The median time from symptom onset to PCI was 3.7 (2.45.6) hours. Primary PCI was performed within 6 hours after symptoms onset in $78 \%$ of cases. Angiographic findings and interventional procedure are detailed in table 4 . The transradial

Table 4 Angiographic characteristics and procedural information

\begin{tabular}{|c|c|}
\hline Procedural findings & \\
\hline Time from symptoms to $\mathrm{PCl}$ (hours) & $3.7(2.4-5.6)$ \\
\hline \multicolumn{2}{|l|}{ Catheterisation access (\%) } \\
\hline Radial & 60 \\
\hline Single-vessel coronary disease (\%) & 53 \\
\hline Single-vessel coronary PCI (\%) & 74 \\
\hline \multicolumn{2}{|l|}{ Infarct-related coronary artery (\%) } \\
\hline Left main & 4 \\
\hline Left anterior descending & 41 \\
\hline Circumflex & 14 \\
\hline Right & 45 \\
\hline CABG & 3 \\
\hline Thrombus aspiration (\%) & 14 \\
\hline \multicolumn{2}{|l|}{ TIMI flow grade after procedure (\%) } \\
\hline 0 & 12 \\
\hline 1 & 1 \\
\hline 2 & 6 \\
\hline 3 & 81 \\
\hline \multicolumn{2}{|l|}{ Coronary stenting (\%) } \\
\hline BMS & 75 \\
\hline DES & 9 \\
\hline POBA & 10 \\
\hline \multicolumn{2}{|l|}{ Procedure success (\%) } \\
\hline Successful PCI & 86 \\
\hline Failed PCI & 11 \\
\hline Complicated PCI & 3 \\
\hline Use of protection device (\%) & 2 \\
\hline $\operatorname{IABP}(\%)$ & 0 \\
\hline Use of inotropes during procedure (\%) & 26 \\
\hline
\end{tabular}

approach was feasible in the majority (60\%) but varied between centres from $40 \%$ to $84 \%$. Successful coronary revascularisation was obtained in $94 \%$ of the patients with an overall successful procedure rate of $86 \%$. Failed PCI occurred in $11 \%$ of the patients, mostly resulting from distal embolisation or coronary dissection or inability to pass the wire through the thrombus. Complicated PCI was observed in 3\% of the patients, mostly resulting from cardiac arrest during primary PCI, either due to refractory cardiogenic shock, refractory ventricular fibrillation or asystole. This was due to left main coronary rupture during angioplasty in one case and to no reflow in another one. During procedure, $26 \%$ of the patients received inotropic support, mainly with dobutamine, but none had intra-aortic counterpulsation support.

\section{Clinical outcomes}

The median hospital stay was 4.0 (2.0-6.5) days (extreme 1-28 days) with coronary care unit stay not prolonged over 3 days. Longer hospital stay was mostly due to social issues. Very short hospital stays ( $<2$ days) resulted in transfers back to the originating cardiology units from where patients were first referred. In-hospital outcomes are shown in table 5.

Of significance, despite intensive antiplatelet and anticoagulant therapy, major or clinically relevant bleeding events were recorded in only six patients (4\%) during in-hospital follow-up. Ventricular arrhythmias occurred in $17 \%$ of patients, all within 24 hours of procedure. Acute renal failure complicated the interventional procedure in $10 \%$ with favourable resolution in half.

The overall in-hospital mortality was $24 \%$. Patients with Killip class I, II, III and IV at admission experimented in-hospital mortality of $12.2 \%, 11.8 \%, 26.7 \%$ and $47.8 \%$, respectively.

\section{Pharmacological management at discharge and follow-up}

At discharge, all but one patient were taking aspirin with $94 \%$ on dual antiplatelet therapy. No platelet function assessment or genetic tests were performed in these patients. The pharmacological management of patients is presented in table 6 .

Complete follow-up was obtained at 6 and 12 months, but $26 \%$ of the patients were lost to follow-up at 24 months. In total, 6-month, 1-year and 2-year overall survival rates were $61 \%$ (95\% CI $53 \%$ to $69 \%$ ), $53 \%$ (45\% to $61 \%$ ) and $43 \%$ (34\% to $52 \%$ ), respectively (figure 1). Almost all (92\%) cardiovascular deaths occurred before day 30 in contrast to noncardiovascular deaths. Two-year survival was significantly lower in females versus males $(\mathrm{p}<0.05)$ and in patients with anterior STEMI $(p<0.01)$ (figure 2$)$. Lower survival in females remained after adjustment on age and centre (Hazard Ratio vs males 1.72, $95 \%$ CI 1.08 to 2.77 ; $<<0.03$ ).

Table 5 In-hospital outcomes

Death (\%) 24

Recurrent MI (\%)

Target vessel revascularisation (\%)

Ventricular arrhythmias (\%)

Major or clinically relevant bleeding (\%)

Acute renal failure (\%)

Stroke (\%)

MI, myocardial infarction 
Table 6 Treatment at hospital discharge

\begin{tabular}{lr}
\hline Aspirin (\%) & 99 \\
Clopidogrel (\%) & 94 \\
Prasugrel (\%) & 3 \\
PPI (\%) & 79 \\
Beta-blockers (\%) & 66 \\
Statins (\%) & 81 \\
CEl or ARA (\%) & 62 \\
Aldosterone inhibitors (\%) & 0 \\
Vitamin K antagonist (\%) & 21 \\
Amiodarone (\%) & 21 \\
\hline ARA, angiotensin receptors antagonists; CEl, converting enzyme inhibitors; PPI, & \\
proton pump inhibitors. &
\end{tabular}

\section{DISCUSSION}

Previous studies have highlighted the increased rate of procedural failure and complications when performing PCI in very old patients, ${ }^{923}$ and data consistently report age to be a strong predictor of mortality. This is particularly high in nonagenarians at 30 days and 1 year and increases with the extent of coronary artery disease and associated conditions. ${ }^{1-4}$ Nevertheless, the results of this study of contemporary practice show that selected nonagenarians presenting with STEMI can be successfully and safely treated by primary PCI.
This study provides interesting data on the characteristics of highly selected cohort. The clinical presentation of an acute myocardial infarction in this specific population may be very different from that seen in younger patients, ranging from completely asymptomatic to general weakness or various thoracic, digestive or neurological symptoms. In our series, all patients presented with thoracic symptoms (acute chest pain and/or shortness of breath), which may be due to a selection bias. For all that, physicians should be aware of atypical and non-specific symptoms, especially abdominal symptoms such as nausea, vomiting, abdominal pain and diarrhoea, weakness and altered mental status. As expected in this population, the majority in our cohort had multivessel coronary disease and mild-to-severe altered left ventricular dysfunction. The prevalence of presentation in Killip class III and IV was high, $>30 \%$ in our series. This figure may be higher as a number of this population may have not been identified as Killip class IV at admission due to the absence of hypotension and the paucity of clinical symptoms as hypoperfusion may be difficult to diagnose in the elderly.

The first point to discuss is the unexpectedly low rate of complications in our series. Although PCI was found to be associated with a higher rate of complications and mortality in patients aged $>75$ years in other studies, ${ }^{24-26}$ we noticed acceptable rates of in-hospital major complications, especially regarding stroke or major bleeding complications with low incidence of acute renal failure. Despite intensive antiplatelet
Figure 1 Kaplan-Meier curves of survival after primary percutaneous coronary intervention for overall mortality (A) and 1-year cardiovascular (B) and non-cardiovascular (C) mortality.

A

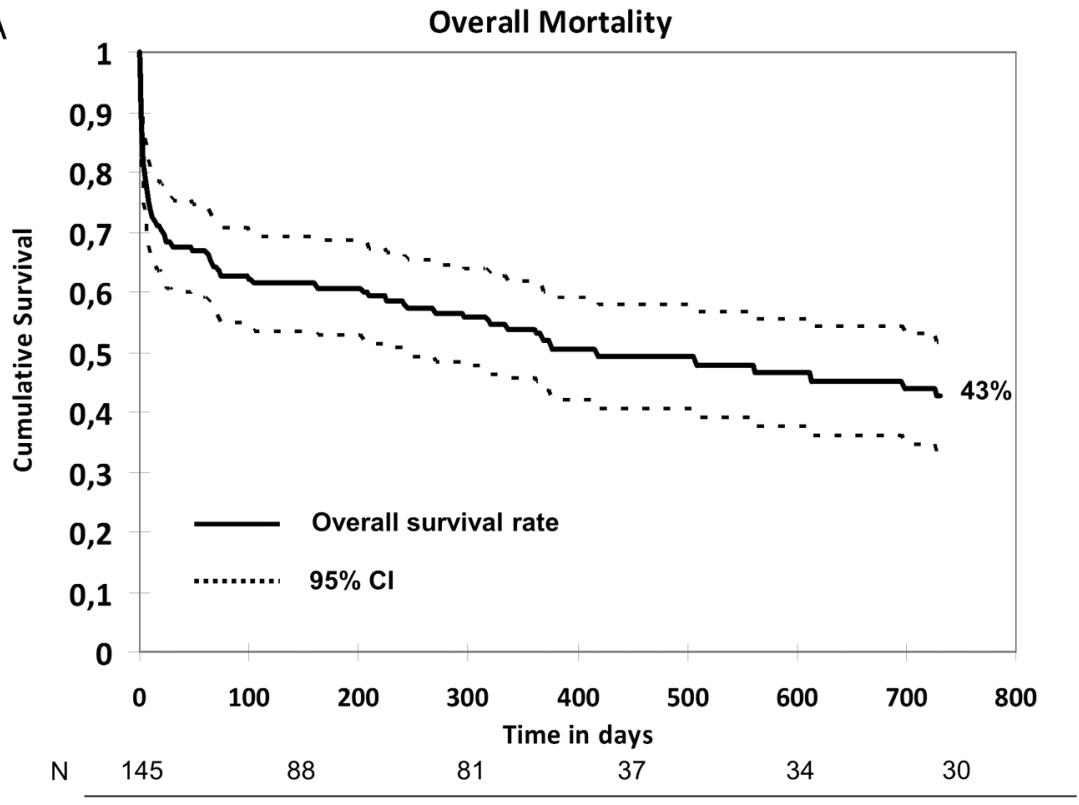

B

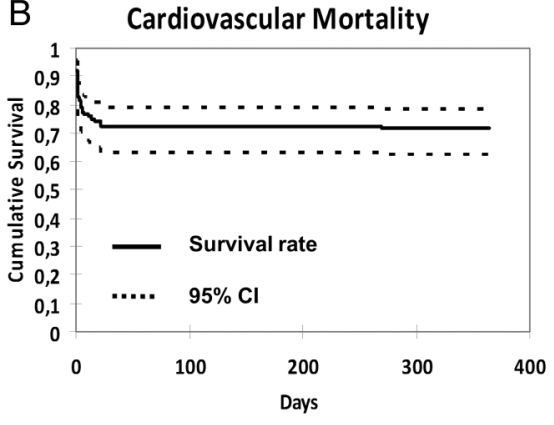

C Non-Cardiovascular Mortality

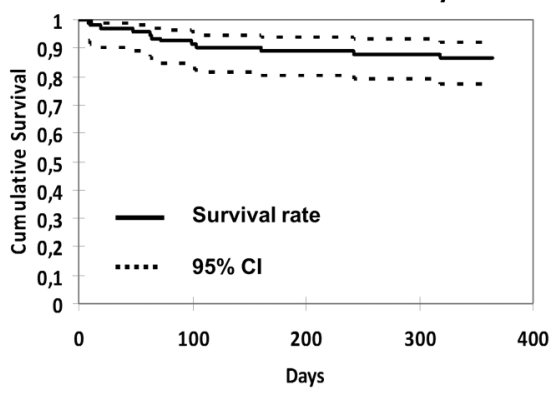

N $\quad 124$

79

$\begin{array}{llll}78 & 30 & \text { N } & 124\end{array}$

82

79

78

30 

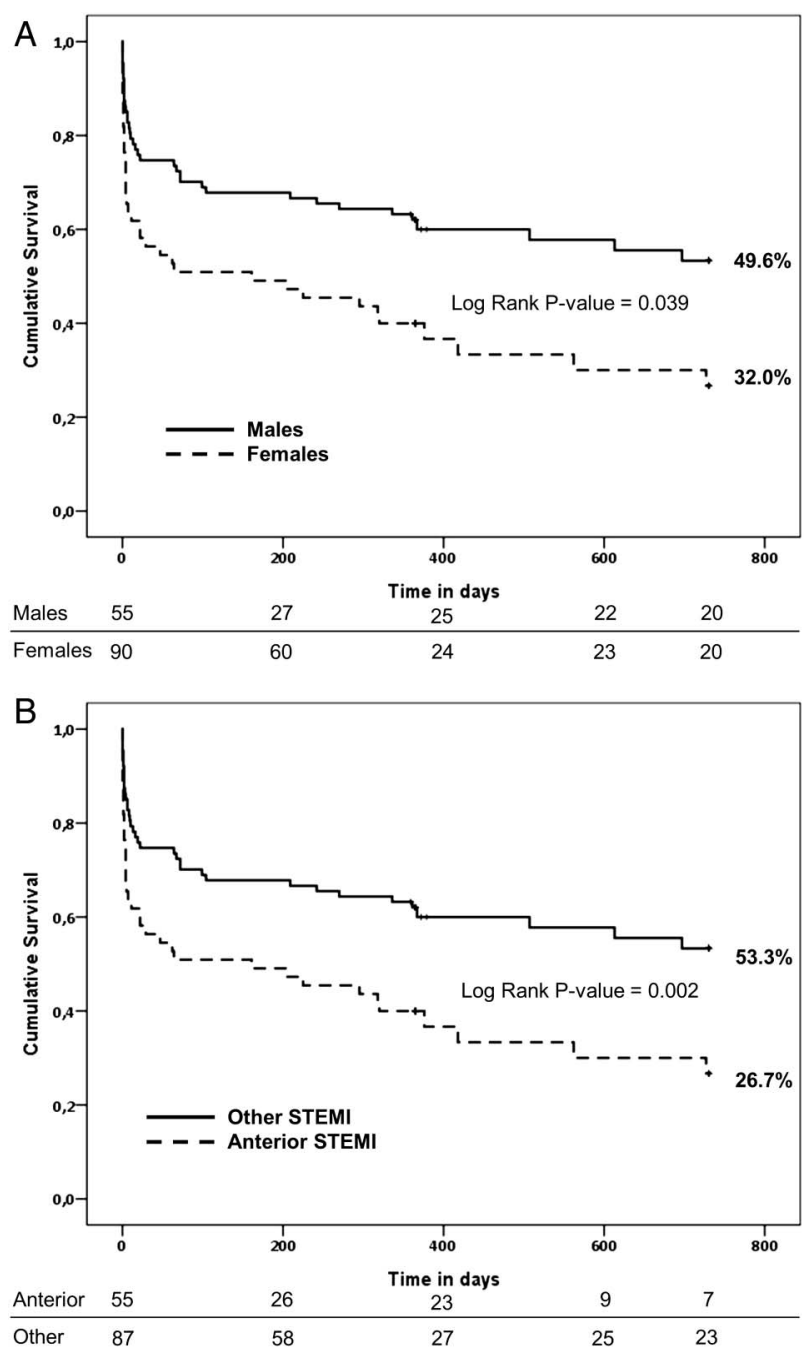

Figure 2 Kaplan-Meier curves showing survival in males versus females $(A)$ and in patients with anterior versus non-anterior ST-segment elevation myocardial infarction (STEMI) (B).

therapy including use of glycoprotein IIb/IIIa inhibitors and most often $600 \mathrm{mg}$ clopidogrel initial loading dose, no fatal or severely disabling bleeding such as intracranial haemorrhage was reported in-hospital. The high rate of procedures conducted through the transradial approach may explain the low rate of access site bleeding complications. Of the six patients who had clinically relevant bleeding, only one received glycoprotein IIbIIIa inhibitors and two underwent femoral access procedures. The very old patients are also at increased risk of contrast-induced acute renal injury and acute renal failure. Indeed, primary PCI with iodinated contrast agents increases the risk of contrast-induced nephropathy exacerbated by altered renal clearance at admission and the frequent Killip III/IV presentation with associated organ hypoperfusion. However, even though the creatinine clearance may have dropped in some patients during hospital stay, this alteration of renal function was observed to be transient except in those with fatal outcome and it is important to note that no death resulted from a renalrelated cause.

The second point of discussion is the antithrombotic strategy. A significant administration of low-molecular-weight heparin was observed in some recruiting centres and is consistent with its benefit over unfractionated heparin previously reported in other studies. ${ }^{27}$ However, its role in the oldest old can be questioned as mean creatinine clearance at admission approaches $30 \mathrm{~mL} / \mathrm{min}$. Furthermore, the choice of the antiplatelet therapy should be carefully made. Although no early bleeding occurred in our series, to date, data remain consistent for clopidogrel prescription over any other thienopyridine, which could increase the long-term bleeding risk. It must be remembered that in some cases oral administration of clopidogrel could be compromised or delayed as a high proportion of the patients may be unable to swallow anything in the context of pulmonary oedema or cardiogenic shock and altered conscious state. Nevertheless, bleeding complications may have occurred after hospital discharge. Prasugrel was rarely administered due to the time period of inclusion and advanced age population. However, new P2Y12 inhibitors appear as an interesting alternative to clopidogrel in selected patients. ${ }^{28}$ Further data may shortly be available in this field (SENIOR study, NCT 02099617).

The third point of discussion is the delay of primary PCI. We found a mean delay from symptoms to primary PCI at 5.8 \pm 7.6 hours, which is a consequence of multiple factors including difficulties in diagnosis and sometimes refusal from other healthcare organisations to perform interventional procedures in elderly patients based on outdated data and their own perception of the risk-to-benefit ratio. The recognition of the benefits of primary PCI in the oldest old should lead to carers and public health measures, recognising that the earlier the diagnosis is made and the patient referred to the catheterisation laboratory, the better will be the outcome from primary PCI. Considering this last argument, additional data provided by our study may help to change current perceptions and potentially influence guidelines on the management of STEMI. ${ }^{29}$

The fourth point of discussion is the global short-term and long-term outcomes. Survival rate at hospital discharge is consistent with previous studies, thus making the case for primary PCI in selected nonagenarian patients presenting with STEMI. Helft $e t$ al recently reported a $24.9 \%$ mortality rate in a cohort of 418 nonagenarians with STEMI and in-hospital follow-up only, very close to the $24 \%$ mortality rate that we noticed. In our series, as in both registries authored by Danzi et $a l^{18}$ and Rigattieri et $a l^{21}$ 6-month survival rates looked similar (61\%, $67 \%$ and $68 \%$, respectively). In patients who were discharged alive, 1-year cardiovascular mortality was very low (only one cardiovascular death from day 30 through 1-year of follow-up). Survival rates were higher than those reported in nonreperfused elderly STEMI patients (in-hospital and 1-year mortality rates of $53 \%$ and $69 \%$, respectively, in a cohort of 139 octogenarians). ${ }^{14}$ Overall survival was in the range of that in general population aged $>90$ years in France (age-specific annual death rates between ages 90 and 94 years of $15 \%$ in females and $21 \%$ in males). ${ }^{30}$

However, we recognised some limitations to our study. The main limitation of this study is that there is no comparison group, such as nonagenarians with STEMI who were not managed with primary PCI. This may be problematic because the study cohort appears to be highly selected with remarkably low comorbidity profile. As our study is retrospective and nonrandomised, it is not possible to draw definite conclusions over the absolute benefit of primary PCI. The generalisability of the study findings to other very old patients treated at other institutions with perhaps less experienced interventionalists is also uncertain. Another important limitation is that we cannot provide data on functional outcomes following the procedure. The proportion of patients who were able to return to their previous level of functioning remains unknown. The proportion of 
patients who previously lived in the community and subsequently required institutionalisation is lacking as well. An unknown proportion of patients may have had new activities of daily living or instrumental activities of daily living dependencies.

In summary, the developed world is ageing and clinical trials typically exclude elderly patients over the age of 75 years. Following myocardial infarction, the outcomes in the oldest old patients are poor and there are limited data in patients $>90$ years of age. Our data support the use of primary PCI to benefit this specific category of population. Finally, these findings show that in keeping with previous series primary PCI is technically feasible in very old patients, with a lower rate of complications than expected, a lower mortality than expected and successful angiographic and clinical results at short-term and longer follow-up. The transradial approach may be routinely chosen by experienced operators to reduce adverse events, particularly puncture site-related bleeding complications. The data presented from several centres in different countries are the largest series to date with the longest follow-up. Our study provides some evidence that in contemporary practice mechanical reperfusion of STEMI in nonagenarians may improve the in-hospital and long-term outcomes.

\section{CONCLUSION}

This study suggests that primary PCI can be safely and successfully performed in nonagenarians presenting with STEMI through a transradial approach. Similar to younger patients in previous large randomised trials, this invasive strategy is associated with a high rate of achieved reperfusion of the infarct-related artery and low incidence of procedure-related complications in this specific population. Use of contemporary strategies in our series was associated with a lower mortality rate than expected considering the severe clinical presentation, and this may be further improved with earlier patient referral. These results should encourage primary PCI to be offered to selected

\section{Key messages}

\section{What is already known on this subject? Nonagenarians presenting with ST elevation myocardial infarction are a growing population but no randomised clinical trial support invasive management. Percutaneous coronary intervention is associated with a higher rate of complications in the elderly patients. Only a few observational studies based on cohorts with limited follow-up suggest that primary percutaneous angioplasty could improve the outcome.}

\section{What might this study add?}

In this cohort of 145 nonagenarians treated by primary percutaneous intervention, 1 -year survival rate is $53 \%$. Our results are consistent with previous small series of patients, demonstrating feasibility and safety of emergent myocardial revascularisation through transradial access and suggesting improvement of long-term survival compared with medical management.

\section{How might this impact on clinical practice?}

Primary percutaneous coronary intervention may be feasible and safely performed through transradial approach in carefully selected nonagenarians with ST-segment elevation myocardial infarction. nonagenarians with acute myocardial infarction. Further clinical studies involving a larger cohort of elderly patients and newer antithrombotic approaches may help to better define the benefits and risks ratio of primary PCI in nonagenarians with STEMI especially in terms of quality of life.

Acknowledgements The authors thank Dr Nicolas Baron (Centre Hospitalier de Versailles, André Mignot Hospital, Le Chesnay, France) for his precious support in the figures' design.

Competing interests TP was funded by the French Federation of Cardiology fellowship grant.

Ethics approval This study was approved by our Hospital's Institutional Review Board (Pitié Salpêtrière Hospital, UPMC, APHP, Paris, France).

Provenance and peer review Not commissioned; externally peer reviewed.

Open Access This is an Open Access article distributed in accordance with the Creative Commons Attribution Non Commercial (CC BY-NC 4.0) license, which permits others to distribute, remix, adapt, build upon this work non-commercially, and license their derivative works on different terms, provided the original work is properly cited and the use is non-commercial. See: http://creativecommons.org/ licenses/by-nc/4.0/

\section{REFERENCES}

1 Mehta RH, Granger CB, Alexander KP, et al. Reperfusion strategies for acute myocardial infarction in the elderly: benefits and risks. J Am Coll Cardiol 2005:45:471-8.

2 National Center for Health Statistics (US). Health, United States, 2006: with chartbook on trends in the health of Americans. Hyattsville, MD: National Center for Health Statistics (US), 2006. http://www.ncbi.nlm.nih.gov/books/NBK21002/

3 Hochholzer W, Wiviott SD, Antman EM, et al. Predictors of bleeding and time dependence of association of bleeding with mortality: insights from the Trial to Assess Improvement in Therapeutic Outcomes by Optimizing Platelet Inhibition With Prasugrel-Thrombolysis in Myocardial Infarction 38 (TRITON-TIMI 38). Circulation 2011;123:2681-9.

4 Hovanesyan A, Rich MW. Outcomes of acute myocardial infarction in nonagenarians. Am J Cardiol 2008;101:1379-83.

5 Lee PY, Alexander KP, Hammill BG, et al. Representation of elderly persons and women in published randomized trials of acute coronary syndromes. JAMA 2001;286:708-13.

6 Yan ZX, Zhou YJ, Zhao YX, et al. Safety and feasibility of transradial approach for primary percutaneous coronary intervention in elderly patients with acute myocardial infarction. Chin Med J (Engl) 2008;121:782-6.

7 Silvain J, Cayla G, Hulot JS, et al. High on-thienopyridine platelet reactivity in elderly coronary patients: the SENIOR-PLATELET study. Eur Heart J 2012;33:1241-9.

8 Smith LG, Herlitz J, Karlsson T, et al. International comparison of treatment and long-term outcomes for acute myocardial infarction in the elderly: Minneapolis/ St. Paul, MN, USA and Goteborg, Sweden. Eur Heart J 2013;34:3191-7.

9 Batchelor WB, Anstrom KJ, Muhlbaier LH, et al. Contemporary outcome trends in the elderly undergoing percutaneous coronary interventions: results in 7,472 octogenarians. National Cardiovascular Network Collaboration. J Am Coll Cardiol 2000;36:723-30

10 Bach RG, Cannon CP, Weintraub WS, et al. The effect of routine, early invasive management on outcome for elderly patients with non-ST-segment elevation acute coronary syndromes. Ann Intern Med 2004;141:186-95.

11 Skolnick $\mathrm{AH}$, Alexander $\mathrm{KP}$, Chen $\mathrm{AY}$, et al. Characteristics, management, and outcomes of 5,557 patients age $>$ or $=90$ years with acute coronary syndromes: results from the CRUSADE Initiative. J Am Coll Cardiol 2007;49:1790-7.

12 Moreno R, Salazar A, Bañuelos C, et al. Effectiveness of percutaneous coronary interventions in nonagenarians. Am J Cardiol 2004:94:1058-60.

13 Babaev A, Frederick PD, Pasta DJ, et al. Trends in management and outcomes of patients with acute myocardial infarction complicated by cardiogenic shock. JAMA 2005;294:448-54.

14 Wood FO, Leonowicz NA, Vanhecke TE, et al. Mortality in patients with ST-segment elevation myocardial infarction who do not undergo reperfusion. Am J Cardiol 2012;110:509-14.

15 Alexander KP, Newby LK, Armstrong PW, et al. Acute coronary care in the elderly, part II: ST-segment-elevation myocardial infarction: a scientific statement for healthcare professionals from the American Heart Association Council on Clinical Cardiology: in collaboration with the Society of Geriatric Cardiology. Circulation 2007;115:2570-89.

16 Steg PG, James SK, Atar D, et al, Task Force on the management of ST-segment elevation acute myocardial infarction of the European Society of Cardiology (ESC) ESC Guidelines for the management of acute myocardial infarction in patients presenting with ST-segment elevation. Eur Heart J 2012;33:2569-619. 
17 Baracioli LM, Serrano CV Jr, Esteves A, et al. Primary coronary angioplasty in a nonagenarian. Arq Bras Cardiol 2002;78:114-21.

18 Danzi GB, Centola M, Pomidossi GA, et al. Usefulness of primary angioplasty in nonagenarians with acute myocardial infarction. Am J Cardiol 2010;106: 770-3.

19 Ionescu CN, Amuchastegui M, Ionescu S, et al. Treatment and outcomes of nonagenarians with ST-elevation myocardial infarction. J Invasive Cardiol 2010:22:474-8.

20 Salinas $\mathrm{P}$, Galeote G, Martin-Reyes $\mathrm{R}$, et al. Primary percutaneous coronary intervention for ST-segment elevation acute myocardial infarction in nonagenarian patients: results from a Spanish multicentre registry. EuroIntervention 2011;6: $1080-4$.

21 Rigattieri S, Cera M, Sciahbasi A, et al. Primary percutaneous coronary intervention in nonagenarians: six-month outcomes from a single-center registry. I Invasive Cardiol 2013;25:242-5.

22 Helft G, Georges JL, Mouranche X, et al. Outcomes of primary percutaneous coronary interventions in nonagenarians with acute myocardial infarction. Int $J$ Cardiol 2015;192:24-9.

23 Dziewierz A, Siudak Z, Rakowski T, et al. Age-related differences in treatment strategies and clinical outcomes in unselected cohort of patients with ST-segment elevation myocardial infarction transferred for primary angioplasty. I Thromb Thrombolysis 2012;34:214-21.

24 Wennberg DE, Makenka DJ, Sengupta A, et al. Percutaneous transluminal coronary angioplasty in the elderly: epidemiology, clinical risk factors, and in-hospital outcomes. The Northern New England Cardiovascular Disease Study Group. Am Heart J 1999;137(Pt 1):639-45.

25 Klein LW, Block P, Brindis RG, et al. Percutaneous coronary interventions in octogenarians in the American College of Cardiology-National Cardiovascular Data Registry: development of a nomogram predictive of in-hospital mortality. J Am Coll Cardiol 2002;40:394-402.

26 Assali AR, Moustapha A, Sdringola $\mathrm{S}$, et al. The dilemma of success: percutaneous coronary interventions in patients $>$ or $=75$ years of age-successful but associated with higher vascular complications and cardiac mortality. Catheter Cardiovasc Interv 2003:59:195-9.

27 Montalescot G, Zeymer U, Silvain J, et al. Intravenous enoxaparin or unfractionated heparin in primary percutaneous coronary intervention for ST-elevation myocardial infarction: the international randomised open-label ATOLL trial. Lancet 2011;378:693-703.

28 Cayla G, Cuisset T, Silvain J, et al. Platelet function monitoring in elderly patients on prasugrel after stenting for an acute coronary syndrome: design of the randomized antarctic study. Am Heart J 2014;168:674-81.

29 Hamm CW, Bassand JP, Agewall S, et al. ESC Guidelines for the management of acute coronary syndromes in patients presenting without persistent ST-segment elevation: The Task Force for the management of acute coronary syndromes (ACS) in patients presenting without persistent ST-segment elevation of the European Society of Cardiology (ESC). Eur Heart J 2011;32:2999-3054.

30 GHO|By category|Life tables by country - France. WHO. http://apps.who.int/gho/ data/view.main.60580 